\title{
Population Genomics Provides Insights into the Population Structure and Climate-driven Adaptation of Collichthys Lucidus
}

\section{Linlin Zhao}

First Institute of Oceanography, Ministry of Natural Resources

\section{Fangyuan Qu}

First Institute of Oceanography, Ministry of Natural Resources

$\mathrm{Na}$ Song

Ocean University of China

\section{Zhiqiang Han}

Zhejiang Ocean University

\section{Tianxiang Gao}

Zhejiang Ocean University

\section{Zhaohui Zhang ( $\nabla$ zhang@fio.org.cn )}

First Institute of Oceanography, Ministry of Natural Resources

\section{Research Article}

Keywords: Collichthys lucidus, genetic diversity, population structure, local adaptive, population genomics

Posted Date: February 1st, 2021

DOl: https://doi.org/10.21203/rs.3.rs-151802/v1

License: (1) This work is licensed under a Creative Commons Attribution 4.0 International License.

Read Full License 


\section{Population genomics provides insights into the population structure and climate-driven} adaptation of Collichthys lucidus

3

6

7

$$
\text { Linlin Zhao }{ }^{1} \text {, Fangyuan } \mathrm{Qu}^{1}, \mathrm{Na} \text { Song }^{2} \text {, Zhiqiang } \mathrm{Han}^{3} \text {, Tianxiang } \mathrm{Gao}^{3} \text {, }
$$
Zhaohui Zhang ${ }^{\text {* }}$

*Corresponding author: zhang@fio.org.cn

\section{Abstract}

Background: Understanding the genetic structure and local adaptive evolutionary mechanisms of marine organisms is crucial for the conservation and management of biological resources. Collichthys lucidus is an ideal candidate for investigating population differentiation and local adaptation under heterogeneous environmental pressure.

Results: To elucidate the fine-scale genetic structure and local thermal adaptation of $C$. lucidus, we performed restriction site-associated DNA tag sequencing (RAD-seq) of 177 individuals from 8 populations, and a total of 184,708 high-quality single nucleotide polymorphisms (SNPs) were identified. All the results revealed significant population structure with high support for two distinct genetic clusters, namely, the northern group (populations DL, TJ, LYG, NT, ZS, and WZ) and southern group (populations XM and ZH). The genetic diversity of the southern group was evidently lower than that of the northern group, which indicated that the southern group was possibly under climate-driven natural selection. In addition, a total of 314 SNPs were found to be significantly associated with temperature variation. Annotations of temperature-related SNPs suggested that genes involved in material (protein, lipid, and carbohydrate) metabolism and immune responses were critical for adaptation to spatially heterogeneous temperatures in natural C. lucidus populations. 
27 Conclusion: In the context of anthropogenic activities and environmental change, the results of the present population genomic work could make important contributions to the understanding of genetic differentiation and adaptation to changing environments.

30 Keywords: Collichthys lucidus, genetic diversity, population structure, local adaptive, 31 population genomics

\section{Background}

Inferring the genetic diversity, population structure and changing patterns of marine species is critical to the successful management of exploited populations, allowing conservation units to be identified and individuals to be assigned to geographical areas $[1,2]$.

However, assessing the current genetic structure and population connectivity of marine species remains a major challenge [3] because marine species usually have large population sizes and long planktonic larval stages. Moreover, the degree of connectivity between populations is often high due to less obvious geographic barriers in the oceans [4]. These biological characteristics may lead to high levels of genetic diversity and low levels of genetic differentiation between populations, even in marine species with a large distribution range [5]. Additionally, many marine species may have had insufficient time for divergence since the colonization of postglacial habitats [6]. In conclusion, it may be difficult to evaluate the genetic diversity and population structure of marine species, which may affect the reasonable management of these species. Population genetics has a great advantage in correctly explaining the population genetic structure of marine species and exploring its influencing factors. Although previous studies have detected genetic differentiation patterns of marine species at a small spatial scale based on a small number of genetic markers [7], it is undeniable that these limited genetic markers may lead to large deviations in the results. In fact, the deficiency of hierarchical 
analysis is extremely obvious when it is applied to the genetics of large populations with limited genetic markers, even for species with weak migration abilities [8]. Therefore, it is essential to increase the number of genetic markers to help us understand the population structure of marine species in detail. Collichthys lucidus is an ideal candidate for studying the influence of complex geographic features of the Northwestern Pacific (NWP) on the population differentiation of marine species. This species is a short-migratory shallow-sea fish that prefers brackish water in estuaries and has a life cycle including pelagic eggs $[9,10]$. Previous population studies

60 based on mitochondrial DNA segments suggested that changes in sea level during the

61 Pleistocene limited the spread of C. lucidus and promoted the emergence of isolated

62 populations, which ultimately had a significant impact on the systematic geographic pattern of this species $[9,11]$. However, previous studies restricted to neutral genetic markers provide limited insights into the mechanisms of population structure and local adaptation. Population genomics provides powerful genome-wide genotyping methods and holds great promise for population genetic studies, as it can allow the detection of local adaptation under climate-driven pressure [12]. This is the case because population genomics may increase the power and resolution of traditional genetic approaches by increasing the number of variable genome-wide genetic markers. Population genomics can also reveal genetic variation in adaptive traits [13].

In this study, eight $C$. lucidus populations were collected from the China Sea, and

72 RAD-seq was used to identify genome-wide single nucleotide polymorphisms (SNPs) in the species. The genome-wide SNPs were further used to explore the high-resolution population genetic structure and local adaptation mechanism of $C$. lucidus. This study provides insights into the evolutionary history and genetic diversity of $C$. lucidus. The results also provide fundamental information for the management and conservation of $C$. lucidus resources under 
fishing pressure.

\section{Results}

80

\subsection{RAD sequencing and genotyping}

$2,707,124,768$ of which were retained after quality filtering (Table S1). A total of

2,561,795,216 read pairs were properly mapped to the genome for SNP calling (Table S1).

After filtering out the low-quality SNPs, 184,708 SNPs were retained for subsequent population structure analysis.

87

\subsection{Population genetic diversity and structure}

These 184,708 SNPs were applied to calculate the genetic diversity of eight $C$. lucidus populations (Table 1). Estimates of $H_{\mathrm{O}}, H_{\mathrm{E}}$, and $P$ i averages over the $184,708 \mathrm{SNPs}$ varied

91 across the eight $C$. lucidus populations $\left(H_{\mathrm{O}}=0.2353 \sim 0.4147, H_{\mathrm{E}}=0.2279 \sim 0.3486\right.$, and $P \mathrm{i}$

$92=0.2348 \sim 0.3560$ ). Among the eight $C$. lucidus populations, the $\mathrm{TJ}$ and $\mathrm{ZH}$ populations showed the highest and lowest $H_{\mathrm{O}}, H_{\mathrm{E}}$, and $P \mathrm{i}$ values, indicating the highest and lowest population genetic diversity, respectively. All eight $C$. lucidus populations generally had a high percentage of polymorphic loci $(73.4023 \sim 99.4640 \%)$. However, the genetic diversity in the $\mathrm{XM}$ and $\mathrm{ZH}$ populations was relatively low according to all indexes. We also found that the $F_{\text {IS }}$ values of the eight $C$. lucidus populations were low (-0.1477 0.0209), suggesting that each population contained a large number of individuals. Additionally, most of the pairwise $F_{\mathrm{ST}}$ values between the eight $C$. lucidus populations were significant (Table 2),

100 ranging from 0.00087 to 0.16222 . Across all eight $C$. lucidus populations except the XM and

101 ZH populations, the $P \mathrm{i}$ and Tajima's $D$ values of genome-wide SNPs showed similar 
102 fluctuation trends (Figure 1). The negative Tajima's $D$ values indicated natural selection

103 pressure in the $\mathrm{XM}$ and $\mathrm{ZH}$ populations, while balancing selection may have resulted in a

104 large proportion of SNPs with positive Tajima's $D$ values in the other populations.

105

106

Table 1. Genetic diversity statistics of eight $C$. lucidus populations.

\begin{tabular}{cccccccc}
\hline \multirow{2}{*}{ Populations } & $\begin{array}{c}\text { Variant } \\
\text { Sites }\end{array}$ & $\begin{array}{c}\text { \% Polymorphic } \\
\text { Loci }\end{array}$ & $\begin{array}{c}\text { Num } \\
\text { Indiv }\end{array}$ & $H_{\mathrm{O}}$ & $H_{\mathrm{E}}$ & $P \mathrm{i}$ & $F_{\mathrm{IS}}$ \\
\hline DL & 184,708 & 97.4544 & 20.0000 & 0.3460 & 0.3282 & 0.3366 & -0.0221 \\
TJ & 184,708 & 99.1809 & 24.0000 & 0.4147 & 0.3486 & 0.3560 & -0.1419 \\
LYG & 184,708 & 99.1820 & 24.0000 & 0.3353 & 0.3360 & 0.3432 & 0.0209 \\
NT & 184,708 & 99.3254 & 24.0000 & 0.3360 & 0.3358 & 0.3430 & 0.0183 \\
ZS & 184,708 & 99.1554 & 20.0000 & 0.3440 & 0.3339 & 0.3425 & -0.0019 \\
WZ & 184,708 & 99.4640 & 24.0000 & 0.4118 & 0.3437 & 0.3510 & -0.1477 \\
XM & 184,708 & 73.4023 & 24.0000 & 0.3086 & 0.2414 & 0.2465 & -0.1442 \\
ZH & 184,708 & 73.6021 & 17.0000 & 0.2353 & 0.2279 & 0.2348 & 0.0013 \\
\hline
\end{tabular}

107

Table 2. Pairwise $F_{\mathrm{ST}}$ values between eight $C$. lucidus populations.

\begin{tabular}{ccccccccc}
\hline $\begin{array}{c}\text { Population } \\
\text { s }\end{array}$ & DL & TJ & LYG & NT & ZS & WZ & XM & ZH \\
\hline DL & - & $\mathbf{0 . 0 0 0 0}$ & $\mathbf{0 . 0 0 0 0}$ & $\mathbf{0 . 0 0 0 0}$ & $\mathbf{0 . 0 0 0 0}$ & $\mathbf{0 . 0 0 0 0}$ & $\mathbf{0 . 0 0 0 0}$ & $\mathbf{0 . 0 0 0 0}$ \\
& & $\mathbf{0}$ & $\mathbf{0}$ & $\mathbf{0}$ & $\mathbf{0}$ & $\mathbf{0}$ & $\mathbf{0}$ & $\mathbf{0}$ \\
TJ & 0.0169 & & $\mathbf{0 . 0 0 0 0}$ & $\mathbf{0 . 0 0 0 0}$ & $\mathbf{0 . 0 0 0 0}$ & $\mathbf{0 . 0 0 0 0}$ & $\mathbf{0 . 0 0 0 0}$ & $\mathbf{0 . 0 0 0 0}$ \\
& 6 & - & $\mathbf{0}$ & $\mathbf{0}$ & $\mathbf{0}$ & $\mathbf{0}$ & $\mathbf{0}$ & $\mathbf{0}$ \\
LYG & 0.0144 & 0.0026 & & $\mathbf{0 . 1 2 6 1}$ & $\mathbf{0 . 0 0 0 0}$ & $\mathbf{0 . 0 0 0 0}$ & $\mathbf{0 . 0 0 0 0}$ & $\mathbf{0 . 0 0 0 0}$ \\
& 9 & 8 & - & $\mathbf{3}$ & $\mathbf{0}$ & $\mathbf{0}$ & $\mathbf{0}$ & $\mathbf{0}$ \\
NT & 0.0169 & 0.0026 & 0.0008 & - & $\mathbf{0 . 0 0 0 0}$ & $\mathbf{0 . 0 0 0 0}$ & $\mathbf{0 . 0 0 0 0}$ & $\mathbf{0 . 0 0 0 0}$ \\
& 3 & 2 & 7 & - & $\mathbf{0}$ & $\mathbf{0}$ & $\mathbf{0}$ & $\mathbf{0}$ \\
ZS & 0.0255 & 0.0062 & 0.0059 & 0.0058 & - & $\mathbf{0 . 0 0 0 0}$ & $\mathbf{0 . 0 0 0 0}$ & $\mathbf{0 . 0 0 0 0}$ \\
& 4 & 8 & 8 & 3 & -0 & $\mathbf{0}$ & $\mathbf{0}$ & $\mathbf{0}$ \\
WZ & 0.0271 & 0.0060 & 0.0068 & 0.0047 & 0.0043 & - & $\mathbf{0 . 0 0 0 0}$ & $\mathbf{0 . 0 0 0 0}$ \\
& 7 & 1 & 1 & 7 & 4 & - & $\mathbf{0}$ & $\mathbf{0}$ \\
XM & 0.1622 & 0.1422 & 0.1443 & 0.1408 & 0.1412 & 0.1359 & - & $\mathbf{0 . 0 0 0 0}$ \\
& 2 & 5 & 9 & 9 & 4 & 1 & - & $\mathbf{0}$ \\
ZH & 0.1631 & 0.1431 & 0.1453 & 0.1420 & 0.1419 & 0.1369 & 0.0036 & - \\
\hline
\end{tabular}

109 


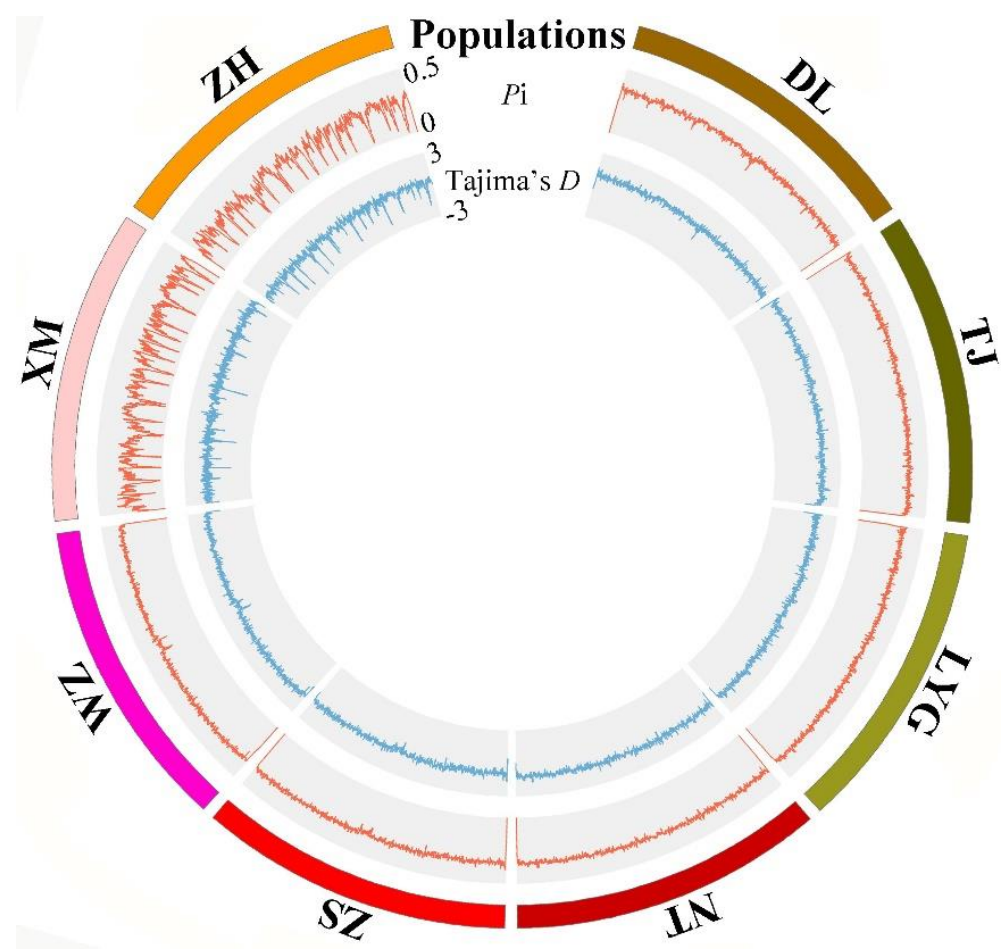

Figure 1. Genome-wide distribution of $P \mathrm{i}$ and Tajima's $D$ values across eight $C$. lucidus populations based on 184,708 SNPs. ADMIXTURE software was first used for clustering analysis of the eight C. lucidus

116 populations (Figure 2). With $K$ values of 2 and 3, the DL, TJ, LYG, NT, ZS, and WZ

117 populations formed an ancestral cluster, and the Xiamen and Zhuhai populations formed

118 another cluster. Meanwhile, the results of the PCA (Figure 3) were consistent with the

119 ADMIXTURE results, which indicated that all populations formed two distinct clusters.

120 NetView $\mathrm{P}$ with $\mathrm{kNN}=20$ was applied to reveal the clustering relationships of all $C$. lucidus

121 individuals at a fine scale, and the results further supported the previous ADMIXTURE

122 clustering pattern with $K=2$ and 3, showing that individuals were grouped into two different

123 clusters, with all individuals from Xiamen and Zhuhai clustered together (Figure 4).

124 Additionally, the hierarchical AMOVA (Table 3) showed that the $F_{\text {ST }}$ across the eight 125 populations was 0.07084 , and there was significant genetic differentiation between the two 126 groups (“Dalian, Tianjin, Lianyungang, Nantong, Zhoushan, and Wenzhou” and "Xiamen and 
129 Table 3. Analysis of molecular variance (AMOVA) performed for two C. lucidus groups

\begin{tabular}{ccccc}
\hline Source of Variation & $\begin{array}{c}\text { Sum of } \\
\text { Squares }\end{array}$ & $\begin{array}{c}\text { Variance } \\
\text { Components }\end{array}$ & $\begin{array}{c}\text { Percentage } \\
\text { Variation }\end{array}$ & Fixation Index \\
\hline Between two groups & 590224.76 & $4353.42 \mathrm{Va}$ & 12.68 & $F_{\mathrm{CT}}=0.13$ \\
$\begin{array}{c}\text { Among populations } \\
\text { within two groups }\end{array}$ & 252582.39 & $331.23 \mathrm{Vb}$ & 0.96 & $F_{\mathrm{SC}}=0.01$ \\
Within eight populations & 5647712.50 & $31907.98 \mathrm{Vc}$ & 93.13 & $F_{\mathrm{ST}}=0.07$ \\
\hline
\end{tabular}

130
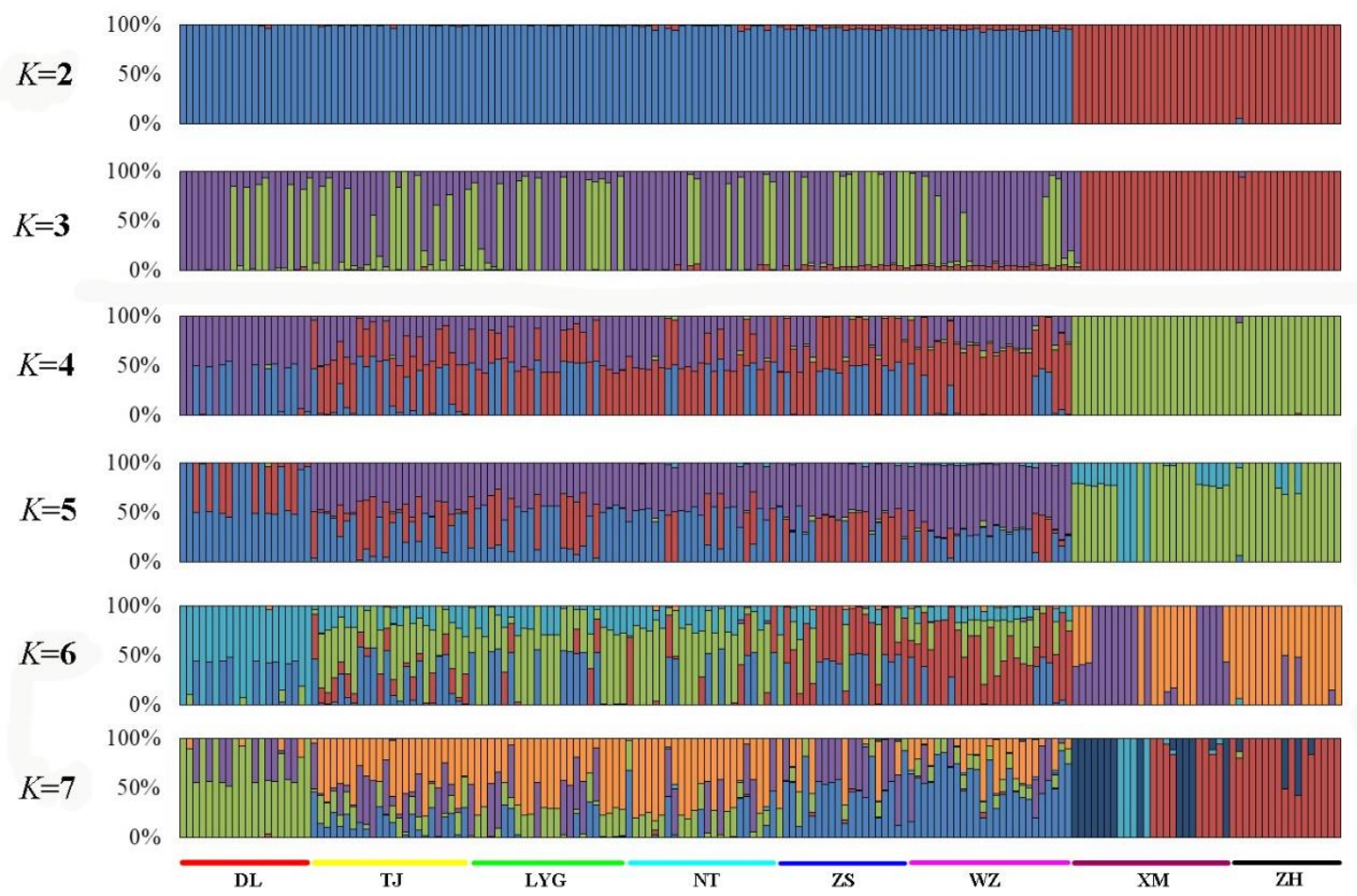

132 Figure 2. Plots of C. lucidus individual ancestry inference for $K=2$ to 7 based on 184,708 


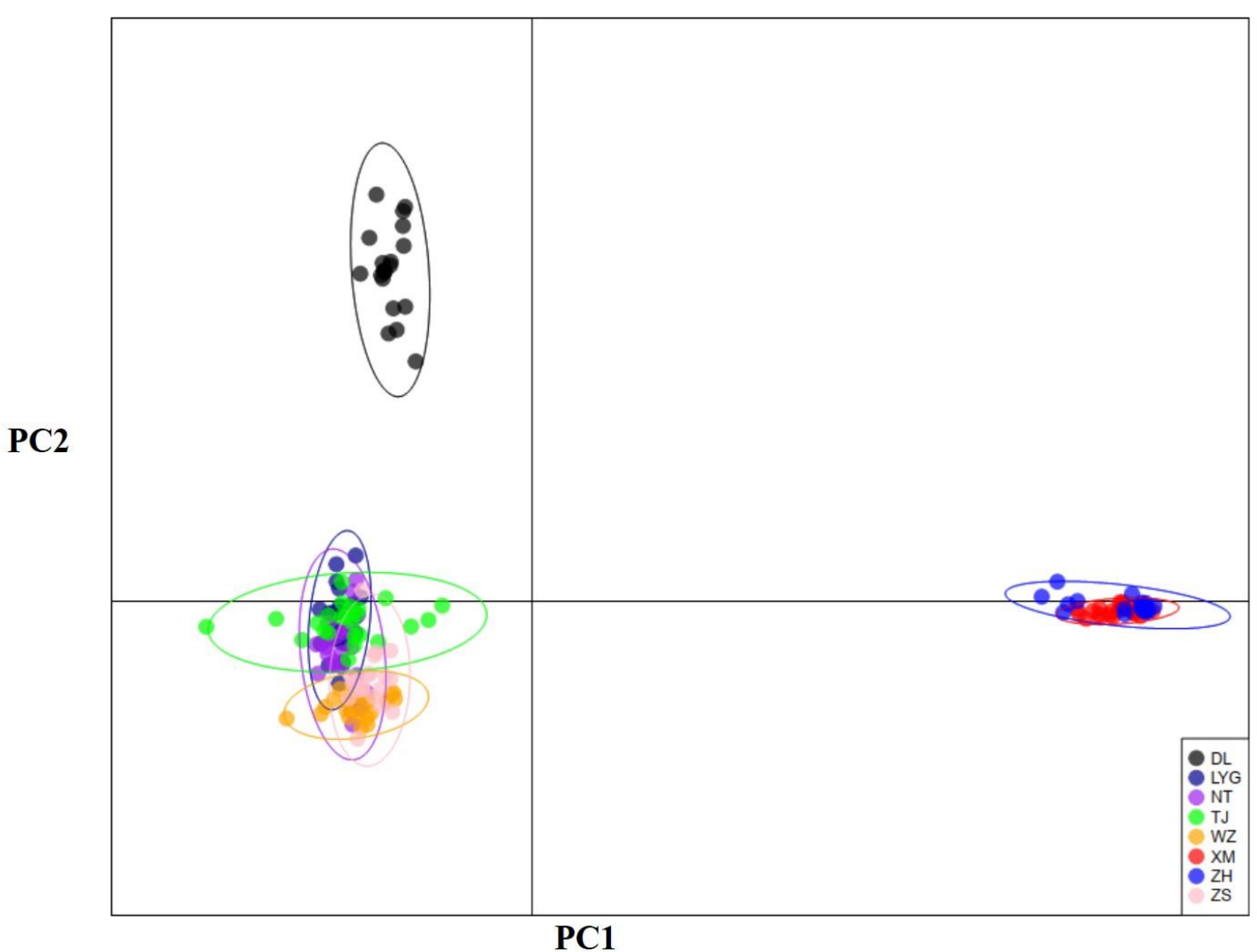

135

Figure 3. PCA scatter plots with prior population information using first and second components. 

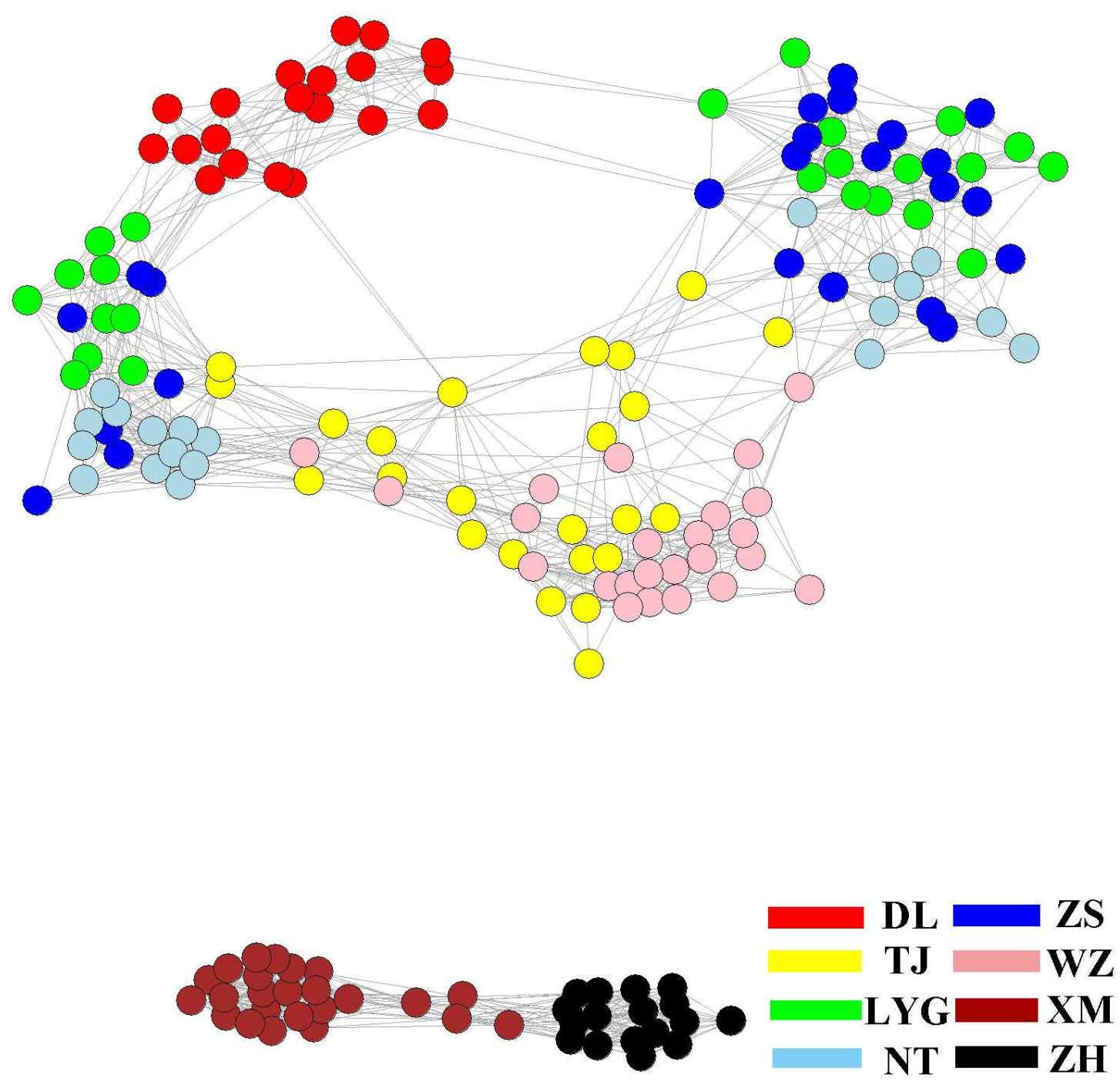

Figure 4. Individual clustering plot based on NetView $\mathrm{P}$ with $\mathrm{KNN}=20$.

\subsection{Candidate genomic regions under temperature-driven selection}

In the present study, we first calculated the average ASST, LSST and HSST of eight sea

144 areas over 68 years (Table S2). Then, Bayenv software revealed a total of 314 SNPs

145 associated with temperature variables. Of these SNPs, 255 were associated with ASST, 56

146 were associated with LSST, and 4 were associated with HSST. There was little overlap

147 among SNPs associated with ASST, LSST and HSST. Thereafter, we used the 314

148 overlapping ASST-related, LSST-related, and HSST-related SNPs as the candidate

149 temperature-selected SNPs. Whole-genome sequences containing 314 SNPs were then used 
150 for further annotations, and the results showed that 105 sequences containing

151 temperature-selected SNPs matched homologous protein sequences in the nonredundant

152 protein sequences $(\mathrm{Nr})$ database (Table S3). Next, the enrichment of sequences containing

153 temperature-selected SNPs in Gene Ontology (GO) categories and Kyoto Encyclopedia of

154 Genes and Genomes (KEGG) pathways was tested, and 30 significantly enriched GO terms

155 (Figure 5) and 16 significantly enriched KEGG pathways (Table S4) were identified.

156

157

158

159

160

161

162

163

164

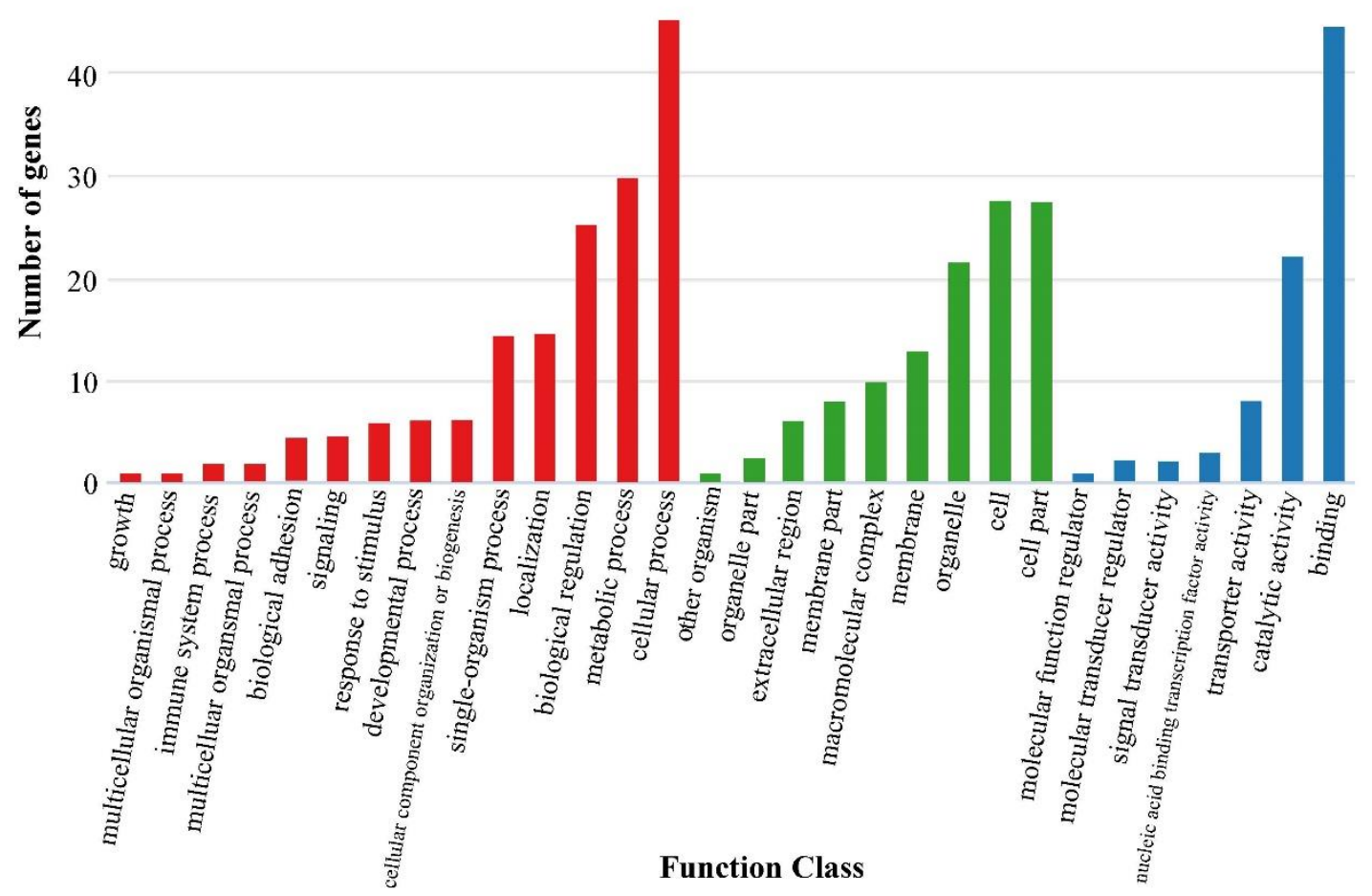

Figure 5. GO annotation information for whole-genome temperature-selected SNPs.

\section{Discussion}

Habitat heterogeneity has profound effects on the population genetic diversity of most marine species. Genome-level analysis not only provides detailed information on the structure, dynamics, and environmental adaptation processes of different populations but also helps us predict how populations will respond to future climate change. We first used RAD-seq to obtain genome-wide SNPs in C. lucidus and then delineated fine population genetic characteristics and local adaptation characteristics of the eight $C$. lucidus populations 
at the genomic level. The present study is helpful in providing a reference for population genomics analysis of other marine species and contributing to the conservation and management of $C$. lucidus, especially in the context of ocean climate change.

3.1 Genome-wide SNPs delineating the fine population structure of the eight C. lucidus populations

Reports of the genetic differentiation of $C$. lucidus are quite limited. The most recent population genetic study was based on the mitochondrial control region [11], which identified C. lucidus in offshore China as being divided into a southern group and a northern group, with Zhoushan as the boundary. Song et al. [9] obtained a contradictory conclusion based on a mitochondrial sequence and suggested that the $C$. lucidus in offshore China could be divided into the East China Sea group and the South China Sea group. Herein, we used 184,708 SNPs to provide a higher-resolution analysis of population structure than the abovementioned studies, and the results supported the conclusion of Song et al. [9]. All the results from ADMIXTURE analysis with $K=2$ and 3, PCA and NetView P showed that the populations investigated in this study were divided into two clusters, in which the DL, TJ, LYG, NT, ZS, and WZ populations formed a cluster and the XM and ZH populations formed another cluster. Notably, C. lucidus is a euryhaline and eurythermal marine fish, and its eggs and adults have a strong diffusion ability. Therefore, this distribution pattern is interesting because there are no obvious barriers between Xiamen and Wenzhou. In

186 fact, the same distribution pattern was also found in Chelon haematocheilus [14]. We speculate that the decline in sea levels during the glacial maximum may have led to long-term geographic isolation between the two C. lucidus clusters [15] and eventually intensified the development of limited dispersal potential, reproductive isolation and local adaptive

190 heterogeneity between the two clusters [9]. During interglacial periods, although rising sea 
191 levels enhanced the dispersal of the two clusters [16, 17], the individuals in the two clusters may not have been able to reproduce, or the diffused individuals may have been unable to adapt to the heterogeneous environment, in turn eventually dying. $F_{\text {ST }}$ values between the two $C$. lucidus clusters were high. Insufficient time to attain migration-drift equilibrium may have resulted in this pattern. Additionally, we speculate that the strong dispersal potential of eggs and adults may be a key driver of the low pairwise $F_{\mathrm{ST}}$ values within $C$. lucidus clusters. In fact, ocean current transport can enhance the diffusion ability of $C$. lucidus and therefore significantly increase gene flow within $C$. lucidus clusters, ultimately contributing to the low pairwise $F_{\mathrm{ST}}$ values within $C$. lucidus clusters [18].

\subsection{Genomic regions of temperature selection in eight C. lucidus populations}

With the advantages of identifying adaptive SNPs, RAD-seq can facilitate insights into the genetics of local adaptation in natural populations [19]. The C. lucidus samples in this study were collected from eight different geographical locations that differed in temperature. Therefore, temperature may be an important selective force affecting the genotypic and phenotypic compositions of local populations. In the present study, we detected a number of important candidate SNPs that appeared to be affected by temperature heterogeneity. Notably, different numbers of candidate SNPs were obtained using different datasets (ASST, LSST, and HSST). We speculate that the LSST is more likely than the HSST to affect the spatial

211 distribution of C. lucidus.

213 temperature-selected SNPs were mainly involved in metabolic and cellular processes, and

214 their functions were mainly in binding and catalytic activity. This suggests that temperature

215 differences drove adaptive differentiation in parts of the genome of $C$. lucidus populations, 
216 ultimately leading to differences in physiological regulation. The KEGG annotation results

217 showed that the sequences containing temperature-selected SNPs were mainly associated

218 with material (protein, lipid, and carbohydrate) metabolism and immune responses. A

219 previous study revealed that the metabolic capacity of organisms was more susceptible to

220 selection under selective force [20]. Moreover, proteins, lipids, and carbohydrates are critical

221 energy materials [21]. Additionally, Lou et al. [22] hypothesized that temperature can affect

222 the priority of metabolic patterns, such as anaerobic carbohydrate metabolism, which is

223 preferred by organisms at low temperatures. Therefore, we speculate that mutations in

224 material metabolism-related genes may cause different $C$. lucidus populations to change their

225 metabolic patterns in order to maintain a maximum metabolic capacity at different

226 temperatures. Additionally, environmental heterogeneity will inevitably cause variation in

227 environmental pressure among $C$. lucidus populations. Mutations in genes associated with the

228 immune response may provide evidence of resistance specificity to temperature stress in

229 different geographic populations. For example, the immune response was shown to be related

230 to local adaptation to different water temperatures in Tylosurus crocodilus crocodilus [23],

231 Trachidermus fasciatus [13], and Larimichthys polyactis [24].

232

\section{4. Conclusion}

234 We revealed the population genetic structure and genomic regions under

235 temperature-driven selection based on genome-wide SNPs in eight $C$. lucidus populations.

236 Genetic structure analysis revealed significant population structure, with high support for two

237 distinct clusters among the eight populations. We speculate that long-term geographic

238 isolation during the glacial maximum may have intensified the development of limited

239 dispersal potential, reproductive isolation and local adaptive heterogeneity between the two $C$.

240 lucidus clusters, eventually leading to strong genetic differentiation. Despite its limited ability 
241 to detect temperature-selected SNPs, RAD-seq remains an effective method for genetic

242 studies of local adaptation to temperature in natural C. lucidus populations. Unsurprisingly,

243 many genetic variants appeared to be selected by temperature differences. Annotations of

244 these temperature-selected SNPs suggested that genes involved in material (protein, lipid, and

245 carbohydrate) metabolism and immune responses were critical for the adaptation of different

246 C. lucidus populations to temperature. This information about the functions of

247 temperature-selected SNPs could help us determine how different C. lucidus populations

248 respond to local temperatures.

250 5. Methods

251

252

\subsection{Specimen collection and RAD sequencing}

All C. lucidus samples were collected from eight locations in China, namely, Dalian

254 (DL), Tianjin (TJ), Lianyungang (LYG), Nantong (NT), Wenzhou (WZ), Zhoushan (ZS),

255 Xiamen (XM), and Zhuhai (ZH) (Figure 6 and Table S5). Muscles were extracted from each

256 individual using sterilized scissors and forceps. All muscle tissues were separately preserved

257 in $95 \%$ ethanol and stored at $-80^{\circ} \mathrm{C}$ prior to the subsequent experiments. Genomic DNA was

258 extracted following the standard phenol-chloroform extraction method. After assessing the

259 quality of the genomic DNA, we constructed a paired-end library with high-quality genomic

260 DNA following the protocol described by Etter et al. [25] and then sequenced the library on

261 the Illumina HiSeq 2500 sequencing platform. 


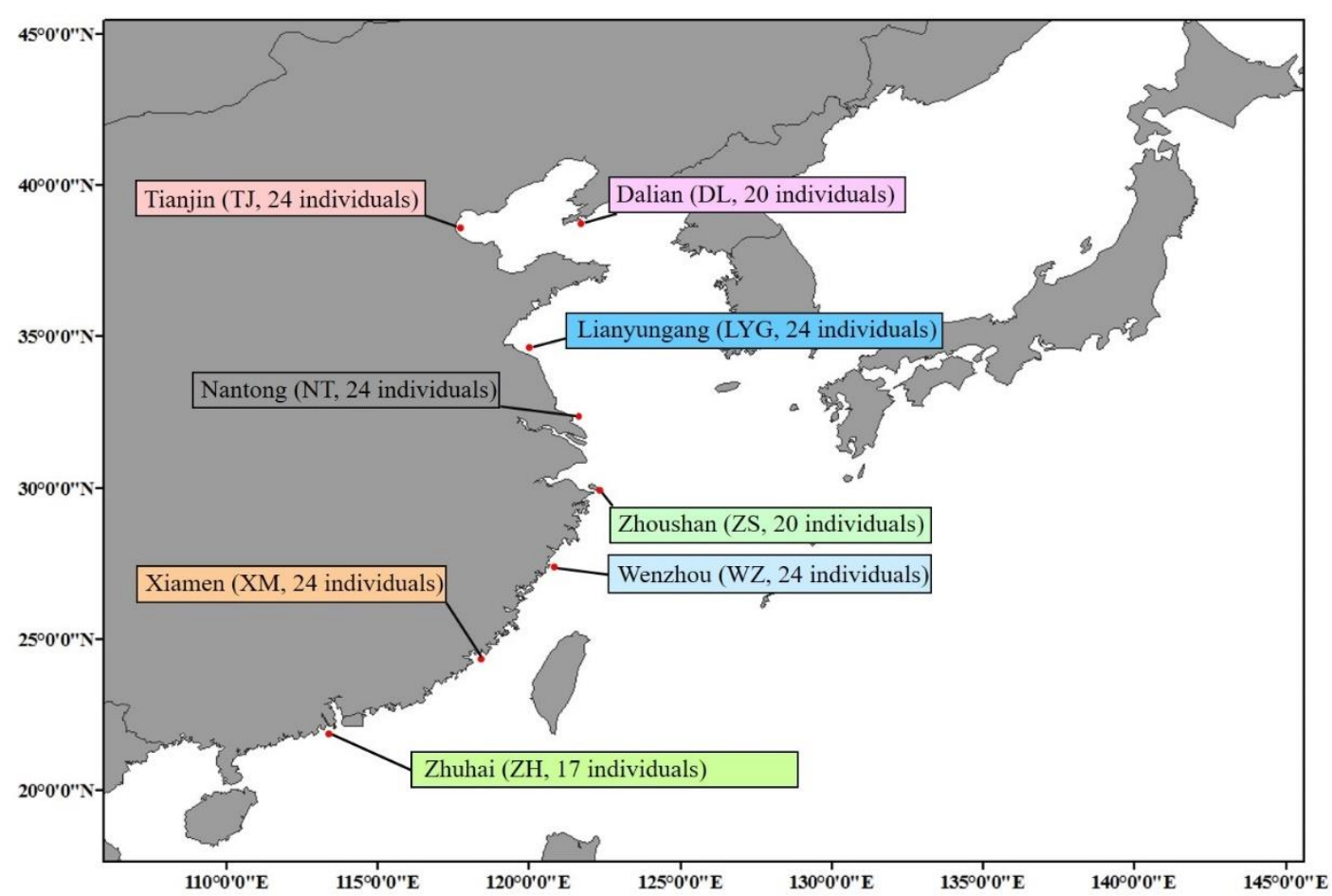

263 Figure 6 Map of the sampling locations and sample numbers for eight $C$. lucidus populations.

\subsection{RAD data processing and SNP filtering}

All raw reads in FASTQ format were filtered using Trimmomatic software (version 0.36;

267 [26]) based on the following criteria: (I) raw reads with sequencing adaptors; (II) a ratio of

268 unidentified nucleotides in the raw reads $\geq 8 \%$; and (III) raw reads that had more than $50 \%$ of

269 base calls with a low quality score $(\mathrm{Q}<30)$. After filtering, we downloaded the whole-genome

270 sequence of C. lucidus [27] and used it as a reference sequence for subsequent SNP filtering.

271 The whole-genome sequence was first constructed into an index file using BWA software

272 (version 0.7.12; [28]). The clean reads of each sample were then aligned to the whole-genome

273 sequence using the "bwa-mem" algorithm in BWA software (version 0.7.12; [28]) with

274 default parameters. SNP calling was subsequently performed using SAMtools software

275 (version 1.3.1; [29]) with the following parameters: -q 1 -C 50 -t AD, ADF, ADR, DP, SP -m

2762 -F 0.002. The generated SNPs were sorted in a variant call format (VCF) file. Furthermore, 
277

278

279

280

281

282

283

284

285

286

287

288

289

we removed the low-quality SNPs using SAMtools (version 1.3.1; [29]) with the following parameters: --maf 0.01 --max-missing 0.1 --min-meanDP 150 --min-alleles 2 --max-alleles 2 --minGQ 98 --minQ 30 --remove-indels --hwe 0.05.

\subsection{Population genetic diversity and differentiation}

The genome-wide patterns of genetic variation, including nucleotide diversity $(P \mathrm{i})$ and Tajima's $D$ at each SNP, were estimated using TASSEL software (version 5.2.31; [30]). The results were visualized using Circos software [31].To calculate the genetic diversity within populations, nucleotide diversity $(P \mathrm{i})$, observed heterozygosity $\left(H_{\mathrm{O}}\right)$, expected heterozygosity $\left(H_{\mathrm{E}}\right)$, and the inbreeding coefficient $\left(F_{\mathrm{IS}}\right)$ were calculated using the "populations" module in Stacks software (version 1.34; [32]. Pairwise genetic differentiation $\left(F_{\mathrm{ST}}\right)$ values between populations and their significance were calculated using Arlequin software (version 3.5; [33] with 10,000 permutations.

Population genetic structure based on all SNPs was analyzed using five methods. (I) The Bayesian model-based clustering program ADMIXTURE (version 1.3.0; [34]) was used to investigate individual ancestries, with five replicates of coancestry cluster $(K)$ values ranging from 2 to 7 . The optimal $K$ value corresponded to the lowest cross-validation error. (II) We conducted a principal component analysis (PCA) using the "adegenet" package [35] implemented in R software to infer population structure. (III) We calculated the allele-sharing distance using PLINK software [36]. The NetView pipeline (version 0.7.1; [37]) with a K-nearest neighbor (KNN) step ranging from 1 to 45 was then used to construct the fine-scale relationships between all individuals, and the networks were visualized using Cytoscape software [38]. (IV) On the basis of the optimal value of $K$, we divided all the groups into two groups (group 1: DL, TJ, LYG, NT, ZS, and WZ; group 2: XM and ZH) and then performed 
an analysis of molecular variance (AMOVA) in Arlequin software (version 3.5; [33]) to estimate the differentiation among groups $\left(F_{\mathrm{CT}}\right)$ and the differentiation among populations within groups $\left(F_{\mathrm{SC}}\right)$.

\subsection{Outlier SNP detection and annotation}

The genotype-environment association method implemented in Bayenv (version 2.0; [39]) was applied to detect putative SNPs correlated with temperature variations. First, we obtained the high-resolution mean lowest sea surface temperature (LSST), mean annual sea surface temperature (ASST) and mean highest sea surface temperature (HSST) data of eight

311 sea areas over 68 years (from 1950 to 2017) by combining data from the Japan

312 Meteorological Agency (JMA; http://www.jma.go.jp/jma/index.html), Advanced Very High

313 Resolution Radiometer (AVHRR; http://oceanwatch.pifsc.noaa.gov/thredds/catalog.html) and

314 Geostationary Operational Environmental Satellites (GOES;

315 http://oceanwatch.pifsc.noaa.gov/thredds/catalog.html). Then, Bayenv tests were applied to

316 identify putative SNPs correlated with temperature variations, and a Bayes factor (BF) value

317 higher than 10 was set as the filtering condition for putative SNPs. We repeated the Bayenv

318 analysis four times to avoid false positives, and only the SNPs that were continuously

319 screened were used for subsequent analysis. Thereafter, we used the overlapping

320 ASST-related, LSST-related, and HSST-related SNPs as the candidate temperature-selected

321 SNPs. To determine the genetic mechanisms underlying temperature-related adaptive

322 differentiation between C. lucidus populations, gene sequences containing these SNPs were 323 then annotated using Blast2GO software [40]. 


\section{Supplementary Information}

Table S1. Sequence information for all individuals.

Table S2. The average ASST, LSST and HSST of eight sea areas.

Table S3. Nr annotation information for whole genome containing temperature-selective SNPs.

Table S4. KEGG annotation information for whole genome containing temperature-selective SNPs.

Table S5. Sampling information for eight sites of C. lucidus.

Abbreviations

$H_{\mathrm{O}}$ : Observe heterozygosity, $H_{\mathrm{E}}$ : Expected heterozygosity; Pi: Polymorphism information; $F_{\text {IS }}$ : the inbreeding coefficient; $F_{\mathrm{ST}}$ : Fixation index; PCA: Principle component analysis; kNN: K-nearest neighbor; ASST: Average sea surface temperature; LSST: Low sea surface temperature; HSST: High sea surface temperature.

\section{Acknowledgements}

335 We would like to thank Fangrui Lou for his technical support and Binbin Shan for collecting 336 samples.

\section{Authors' contribution}

Z.Z., Z.L. and G.T. conceived and designed the experiments. Q.F. and S.N. performed the

340 sample DNA extraction. Z.L. and H.Z. performed the computational analyses. Z.L. drafted

341 the manuscript. All authors read and approved the final manuscript. 
344 This work was supported by the National Natural Science Foundation of China (41706187).

346 Availability of data and materials

347 Sequences are available from GenBank with the Bioproject accession numbers

348 PRJNA679902.

\section{Declarations}

351 The authors declare that they have no competing interests.

352

\section{Ethics approval and consent to participate}

C. lucidus is not protected species in China. Tissues used in this study were collected from

wild according to the regulations of Fishery Administration of the Ministry of Agriculture. All

356 experimental protocols and procedures were approved by Institutional Animal Care and Use

357 Committee of First Institute of Oceanography, Ministry of Natural Resources.

358

359 Consent for publication

360 Not applicable

361

362 Author details

$363{ }^{1}$ First Institute of Oceanography, Ministry of Natural Resources, Qingdao, Shandong, 266100, 364 China.

3652 Fishery College, Ocean University of China, Qingdao, Shandong, 266061, China.

$366{ }^{3}$ Fishery College, Zhejiang Ocean University, Zhoushan, Zhejiang, 316022, China.

368 References 
369 1. Funk WC, McKay JK, Hohenlohe PA, Allendorf FW. Harnessing genomics for 370 delineating conservation units. Trends Ecol Evol. 2012;27:489-96.

371 2. Nielsen EE, Cariani A, Mac Aoidh E, Maes GE, Milano I, Ogden R, et al.

372 Gene-associated markers provide tools for tackling illegal fishing and false 373 eco-certification. Nat Commun. 2012;3:851.

374 3. Miller AD, van Rooyen A, Rašić G, Ierodiaconou DA, Gorfine HK, Day R, et al. 375 Contrasting patterns of population connectivity between regions in a commercially 376 important mollusc Haliotis rubra: integrating population genetics, genomics and 377 marine LiDAR data. Mol Ecol. 2016;25:3845-64.

378 4. Allendorf FW, Hohenlohe PA, Luikart G. Genomics and the future of conservation 379 genetics. Nat Rev Genet. 2010;11:697-709.

380 5. DeWoody JA, Avise JC. Microsatellite variation in marine, freshwater and anadromous fishes compared with other animals. J Fish Biol. 2000;56:461-73.

6. Hauser L, Carvalho GR. Paradigm shifts in marine fisheries genetics: ugly hypotheses slain by beautiful facts. Fish Fish. 2008;9:333-62.

384 7. Teske PR, Sandoval-Castillo J, van Sebille E, Waters J, Beheregaray LB. On-shelf larval retention limits population connectivity in a coastal broadcast spawner. Mar Ecol Prog Ser. 2015;532:1-12.

8. Xu S, Song N, Zhao L, Cai S, Han Z, Gao T. Genomic evidence for local adaptation in the ovoviviparous marine fish Sebastiscus marmoratus with a background of population homogeneity. Sci Rep. 2017;7:1562.

390 9. Song N, Ma G, Zhang X, Gao T, Sun D. Genetic structure and historical demography of Collichthys lucidus inferred from mtDNA sequence analysis. Environ Biol Fishes.

393 10. Song W, Jiang KJ, Zhang FY, Zhao M, Ma LB. Molecular cloning and gene 
expression analysis of cystatin C-like proteins in spinyhead croaker Collichthys lucidus. Genet Mol Res. 2016;15. doi: 10.4238/gmr.15017417.

396 11. Liang SZ, Song W, Ma CY, Jiang KJ, Zhang FY, Zhao M, et al. Genetic structure of Collichthys lucidus populations from China coastal waters based on mtDNA control region. Mar Fish. 2019;41:138-48.

12. Lozier JD, Zayed A. Bee conservation in the age of genomics. Conserv Genet. 2017;18:713-29.

401

13. Li YL, Xue DX, Zhang BD, Liu JX. Population genomic signatures of genetic structure and environmental selection in the catadromous roughskin sculpin trachidermus fasciatus. Genome Biol Evol. 2019;11:1751-64.

14. Liu JX, Gao TX, Wu SF, Zhang YP. Pleistocene isolation in the Northwestern Pacific marginal seas and limited dispersal in a marine fish, Chelon haematocheilus (Temminck \& Schlegel, 1845). Mol Ecol. 2007;16:275-88.

15. Palumbi SR. Genetic divergence, reproductive isolation, and marine speciation. Annu Rev Ecol Syst. 1994;25:547-72.

16. Shen KN, Jamandre BW, Hsu CC, Tzeng WN, Durand JD. Plio-Pleistocene sea level and temperature fluctuations in the northwestern Pacific promoted speciation in the globally-distributed flathead mullet Mugil cephalus. BMC Evol Biol. 2011;11:83.

412 17. Xu J, Chan TY, Tsang LM, Chu KH. Phylogeography of the mitten crab Eriocheir sensu stricto in East Asia: pleistocene isolation, population expansion and secondary contact. Mol Phylogenet Evol. 2009;52:45-56.

415 18. Hu ZM, Zhang J, Lopez-Bautista J, Duan DL. Asymmetric genetic exchange in the brown seaweed Sargassum fusiforme (Phaeophyceae) driven by oceanic currents. Mar Biol. 2013;160:1407-14.

418 19. Catchen JM, Hohenlohe PA, Bernatchez L, Funk WC, Andrews KR, Allendorf FW. 
Unbroken: RADseq remains a powerful tool for understanding the genetics of adaptation in natural populations. Mol Ecol Resour. 2017;17:362-5.

20. Priede IG. Metabolic scope in fishes. In: Tytler P and Calow P, editor. Fish Energetics: New Perspective. London: Croom-Helm; 1985.

21. Wang J. Advances in studies on the ecology and reproductive biology of Trachidermus fasciatus Heckel. Acta Hydrobiol Sin. 1999;23:729-34.

22. Lou F, Han Z, Gao T. Transcriptomic responses of two ecologically divergent populations of Japanese Mantis Shrimp (Oratosquilla oratoria) under thermal stress. Animals (Basel). 2019;9:399.

23. Flanagan SP, Rose E, Jones AG. Population genomics reveals multiple drivers of population differentiation in a sex-role-reversed pipefish. Mol Ecol. 2016;25:5043-72.

24. Zhang BD, Xue DX, Li YL, Liu JX. RAD genotyping reveals fine-scale population

25. Etter PD, Preston JL, Bassham S, Cresko WA, Johnson EA. Local de novo assembly of RAD paired-end contigs using short sequencing reads. PLoS One. 2011;6:e18561.

26. Bolger AM, Lohse M, Usadel B. Trimmomatic: a flexible trimmer for Illumina sequence data. Bioinformatics. 2014;30:2114-20.

27. Cai M, Zou Y, Xiao S, Li W, Han Z, Han F, et al. Chromosome assembly of Collichthys lucidus, a fish of Sciaenidae with a multiple sex chromosome system. Sci

28. Li H, Durbin R. Fast and accurate short read alignment with Burrows-Wheeler transform. Bioinformatics. 2009;25:1754-60. alignment/map format and SAMtools. Bioinformatics. 2009;25:2078-9. 
30. Bradbury PJ, Zhang Z, Kroon DE, Casstevens TM, Ramdoss Y, Buckler ES. TASSEL: software for association mapping of complex traits in diverse samples. Bioinformatics. 2007;23:2633-5.

31. Krzywinski M, Schein J, Birol I, Connors J, Gascoyne R, Horsman D, et al. Circos: an information aesthetic for comparative genomics. Genome Res. 2009;19:1639-45.

32. Catchen J, Hohenlohe PA, Bassham S, Amores A, Cresko WA. Stacks: an analysis tool set for population genomics. Mol Ecol. 2013;22:3124-40.

33. Excoffier L, Lischer HE. Arlequin suite ver 3.5: a new series of programs to perform population genetics analyses under Linux and Windows. Mol Ecol Resour.

34. Alexander DH, Novembre J, Lange K. Fast model-based estimation of ancestry in unrelated individuals. Genome Res. 2009;19:1655-64.

35. Jombart T, Devillard S, Balloux F. Discriminant analysis of principal components: a new method for the analysis of genetically structured populations. BMC Genet.

36. Purcell S, Neale B, Todd-Brown K, Thomas L, Ferreira MA, Bender D, et al. PLINK: a tool set for whole-genome association and population-based linkage analyses. Am J Hum Genet. 2007;81:559-75.

37. Steinig EJ, Neuditschko M, Khatkar MS, Raadsma HW, Zenger KR. netview p: a network visualization tool to unravel complex population structure using genome-wide SNPs. Mol Ecol Resour. 2016;16:216-27.

38. Kohl M, Wiese S, Warscheid B. Cytoscape: software for visualization and analysis of biological networks. Methods Mol Biol. 2011;696:291-303.

39. Coop G, Witonsky D, Di Rienzo A, Pritchard JK. Using environmental correlations to identify loci underlying local adaptation. Genetics. 2010;185:1411-23. 
469 40. Conesa A, Götz S, García-Gómez JM, Terol J, Talón M, Robles M. Blast2GO: a universal tool for annotation, visualization and analysis in functional genomics research. Bioinformatics. 2005;21:3674-6. 
Figures

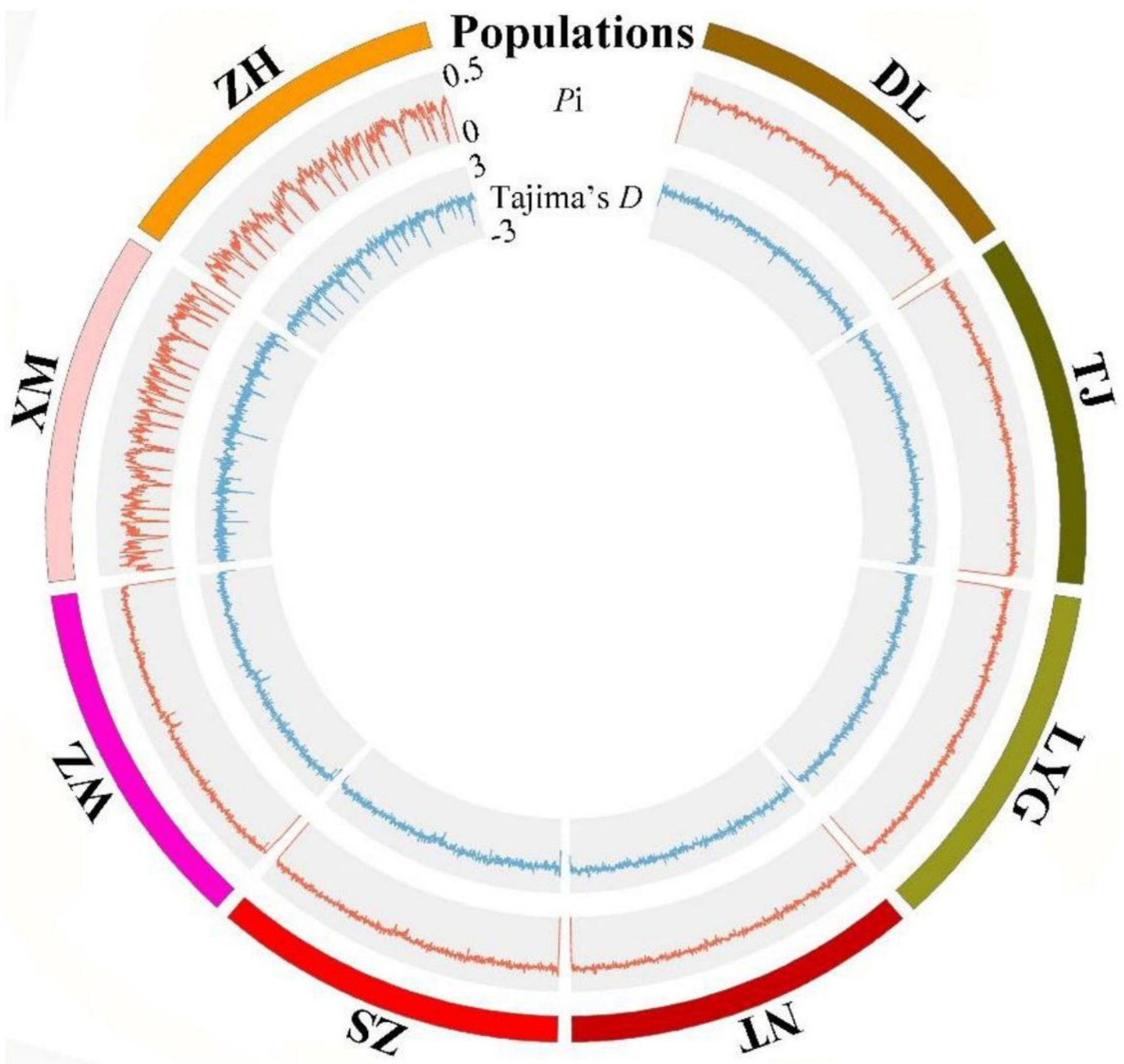

Figure 1

Genome-wide distribution of Pi and Tajima's D values across eight $\mathrm{C}$. lucidus populations based on 184,708 SNPs. 


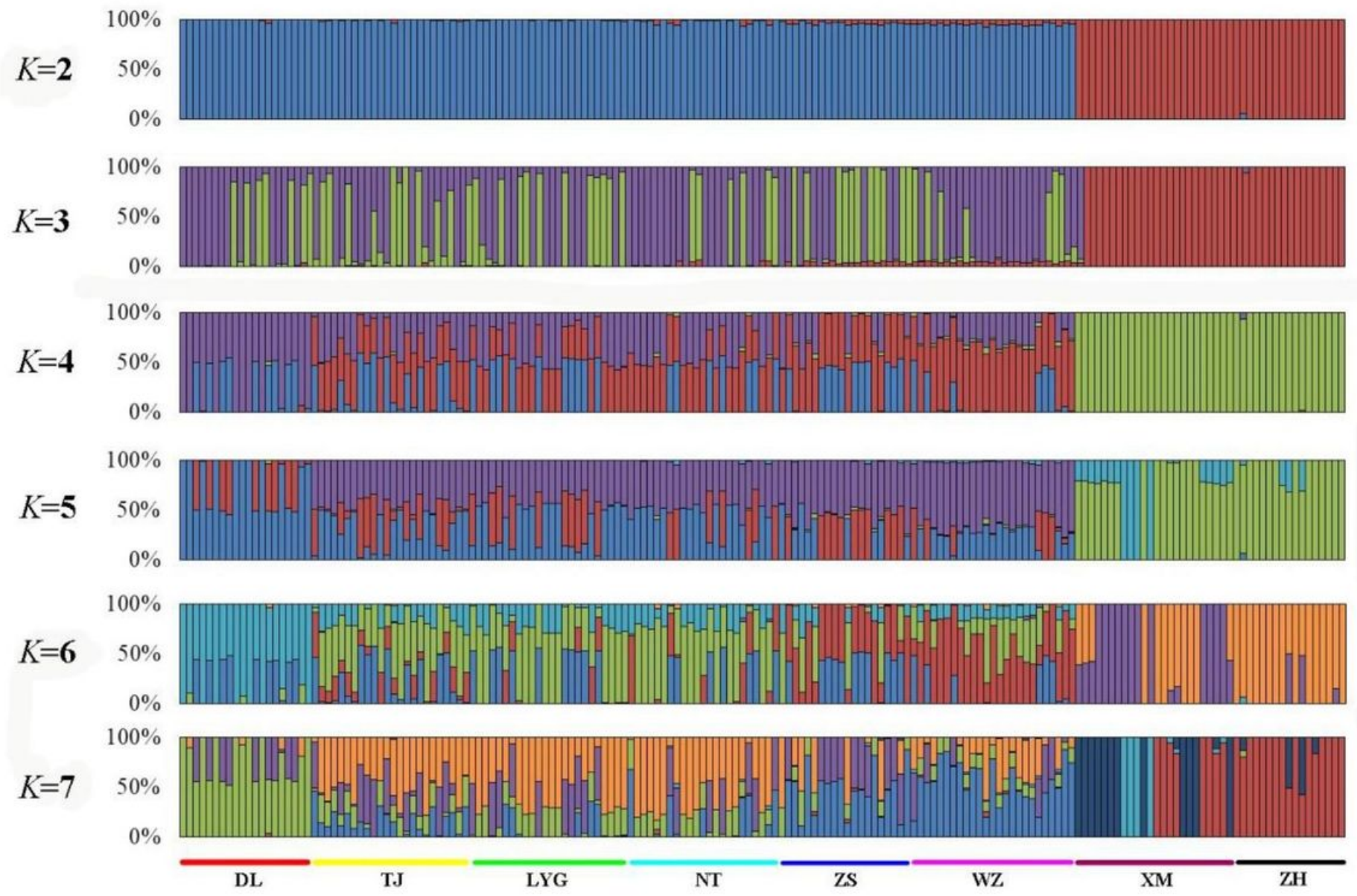

Figure 2

Plots of C. lucidus individual ancestry inference for $K=2$ to 7 based on 184,708 SNPs. 


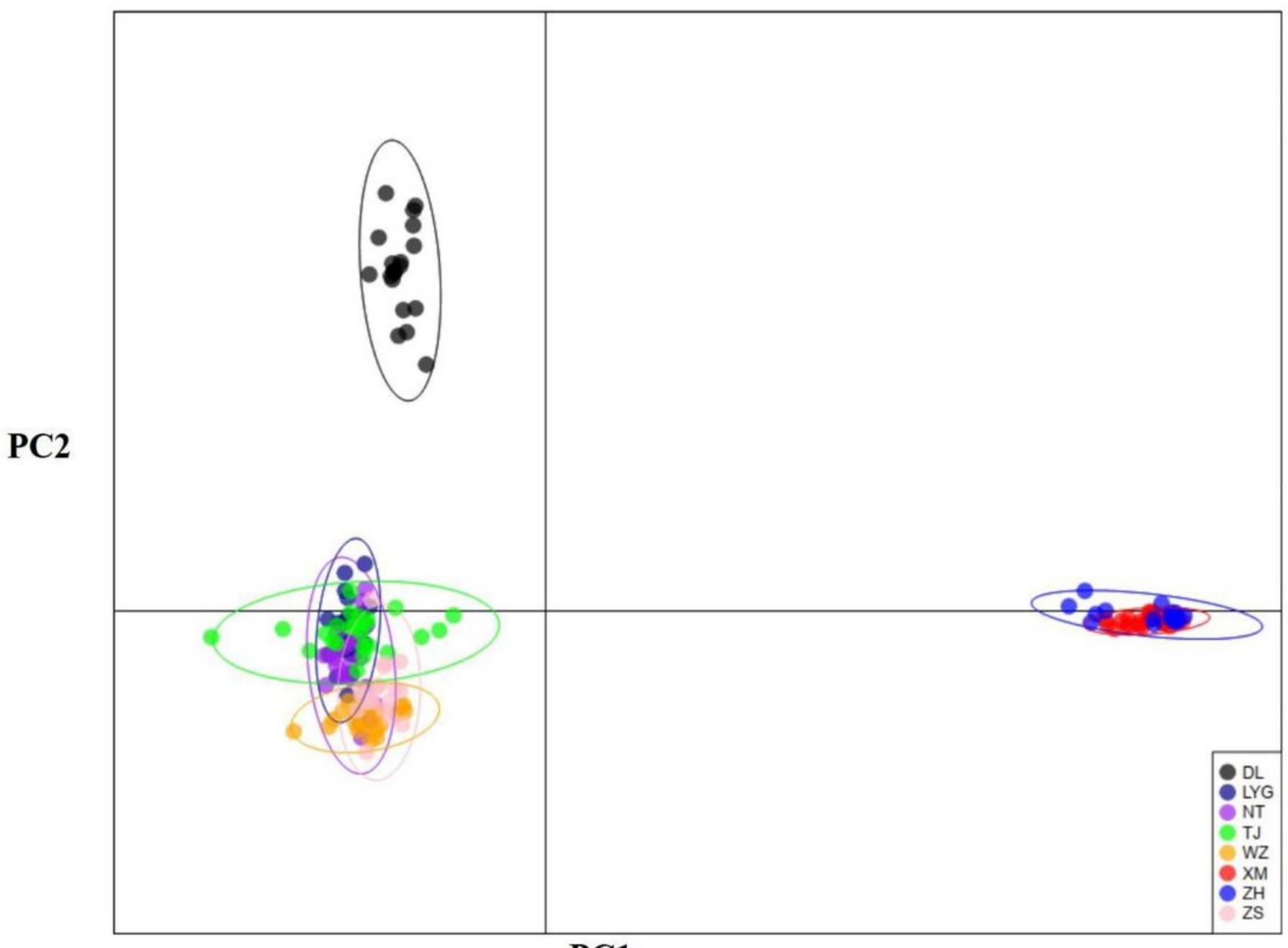

PC1

Figure 3

PCA scatter plots with prior population information using first and second components. 

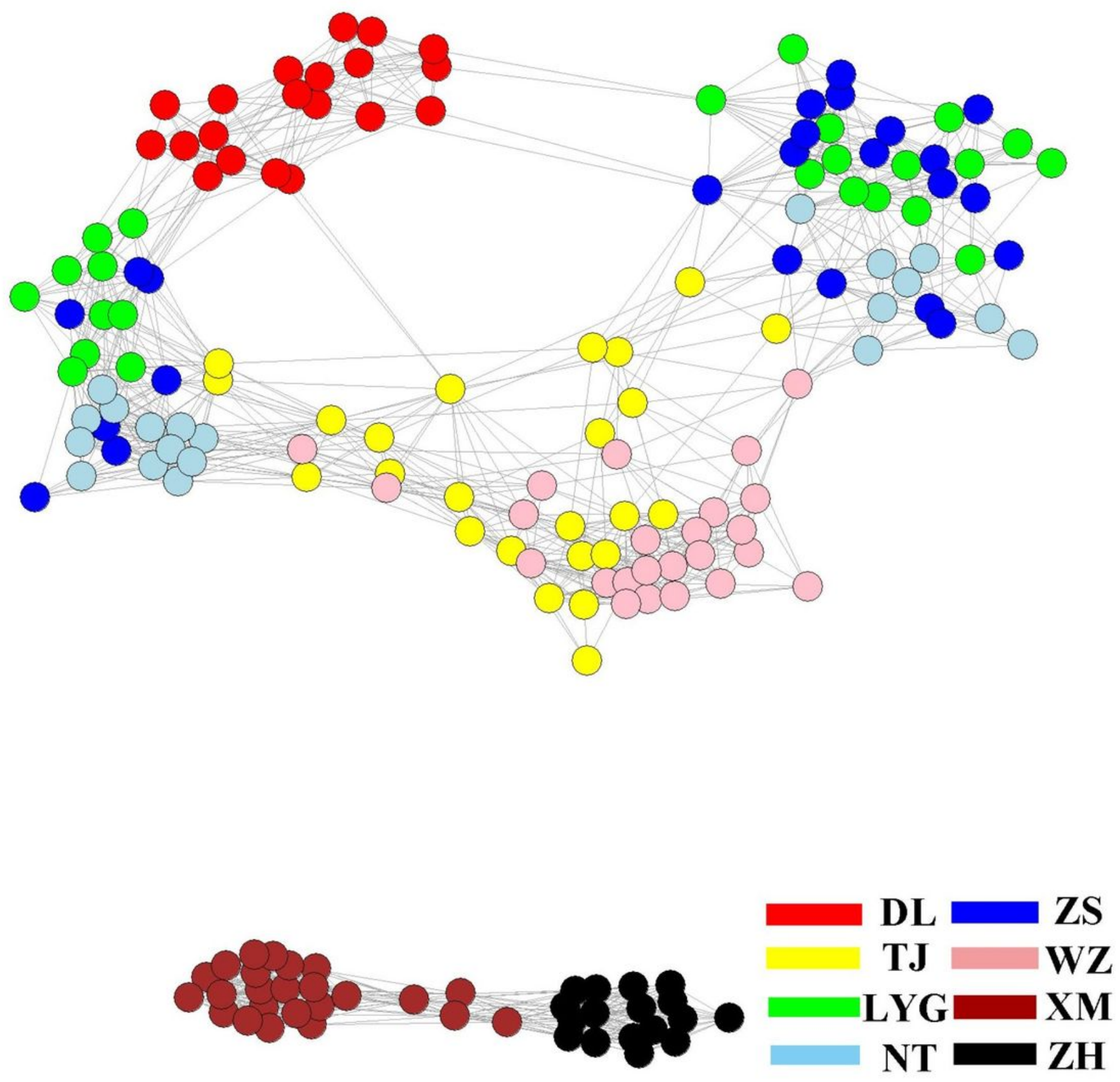

Figure 4

Individual clustering plot based on NetView P with KNN = 20 . 


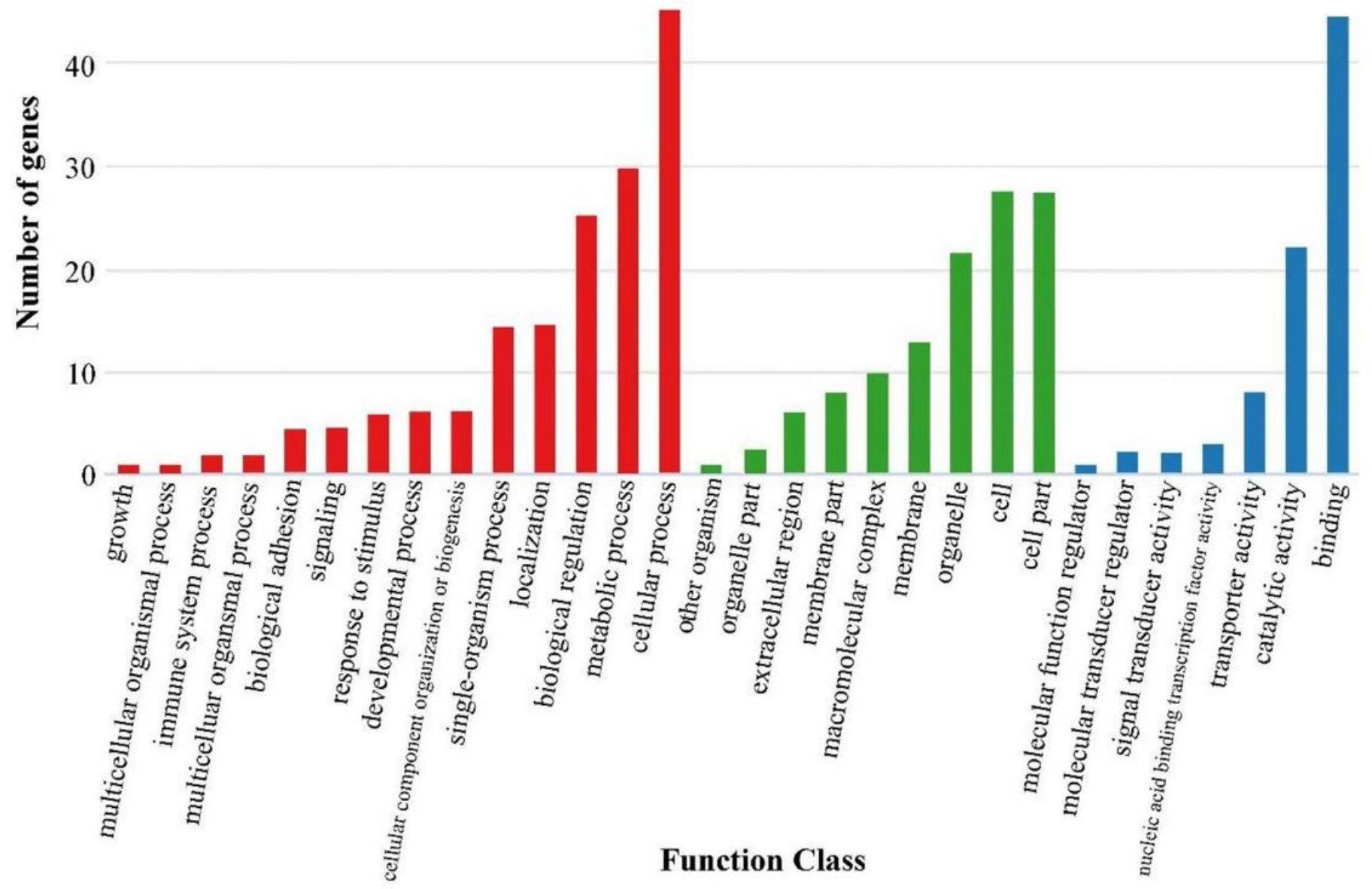

Figure 5

GO annotation information for whole-genome temperature-selected SNPS. 


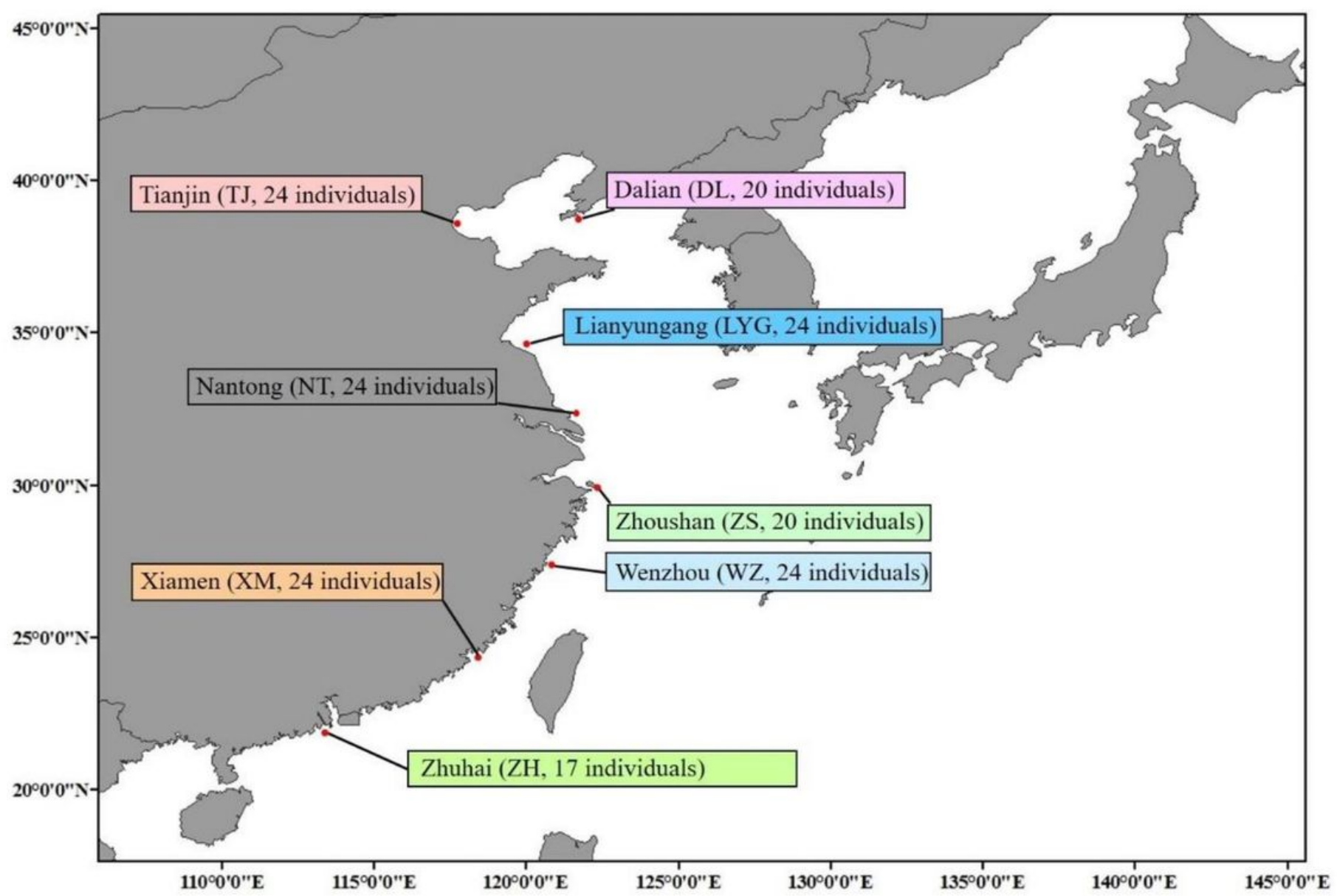

\section{Figure 6}

Map of the sampling locations and sample numbers for eight $\mathrm{C}$. lucidus populations. Note: The designations employed and the presentation of the material on this map do not imply the expression of any opinion whatsoever on the part of Research Square concerning the legal status of any country, territory, city or area or of its authorities, or concerning the delimitation of its frontiers or boundaries. This map has been provided by the authors.

\section{Supplementary Files}

This is a list of supplementary files associated with this preprint. Click to download.

- supplementarymaterial.pdf 